\title{
Dynamics of a Breast Cancer Model for Neutropenia Case due to Chemotherapy Effects
}

\author{
M. Ivan Ariful Fathoni $\mathbb{D}^{1,2}$ Fajar Adi-Kusumo ${ }^{1}{ }^{1},{ }^{1}$ Gunardi Gunardi, ${ }^{1}$ \\ and Susanna Hilda Hutajulu $\mathbb{D}^{3}$ \\ ${ }^{1}$ Department of Mathematics, Faculty of Mathematics and Natural Sciences, Universitas Gadjah Mada, Yogyakarta, Indonesia \\ ${ }^{2}$ Department of Mathematics Education, Faculty of Teacher Training and Education, Universitas Nahdlatul Ulama Sunan Giri, \\ Bojonegoro, Indonesia \\ ${ }^{3}$ Department of Internal Medicine, Faculty of Medicine, Public Health, and Nursing, Universitas Gadjah Mada, \\ Dr. Sardjito General Hospital, Yogyakarta, Indonesia
}

Correspondence should be addressed to Fajar Adi-Kusumo; f_adikusumo@ugm.ac.id

Received 24 July 2021; Accepted 29 September 2021; Published 20 October 2021

Academic Editor: Jianshe Yu

Copyright (c) 2021 M. Ivan Ariful Fathoni et al. This is an open access article distributed under the Creative Commons Attribution License, which permits unrestricted use, distribution, and reproduction in any medium, provided the original work is properly cited.

Breast cancer is a type of carcinoma with a high prevalence. The treatment of breast cancer through chemotherapy can cause a risk to healthy cells throughout the body. The neutrophil is one of the cells that is influenced by chemotherapy drugs. Chemotherapyinduced neutropenia is one of the most common toxic effects experienced by patients and often threatens chemotherapy to use efficiency. In this paper, we introduce an interaction model between blood components, i.e., neutrophil, lymphocytes, and albumin, with chemotherapy drugs. The model is important to understand the neutropenia effect due to chemotherapy in mathematical perspective and to calculate breast cancer patients' survival level. Our model is a four-dimensional system of the first-order ODE with 13-dimensional parameter space. We focus our study for understanding the steady-state conditions and the bifurcations when the parameter values are varied. Here, we also study the role of albumin for reducing the neutropenia effects for breast cancer patients mathematically, where the results can be used as an alternative solution for treating neutropenia in a breast cancer case.

\section{Introduction}

Breast cancer is a type of a high prevalence carcinoma. It can occur in both men and women, where the higher prevalence is in women. Data from WHO in 2018 showed that the number of new breast cancer cases was 2,088,849 or $11.6 \%$ of the world's total number of cancer cases. This number is only different from 5,027 cases of lung cancer, which occupies the first position. Based on this number, 626,679 of them were the death cases. Asia is in the top rank of the number of breast cancer incidence and mortality in the world [1]. The disease is treatable if it is detected earlier and is still in its earler stages. The higher the stage implies, the smaller the life expectancy is. There is no cancer that has a $100 \%$ mortality rate; it means that the treatment can be done to get a cure, especially if treatment is started earlier [2].

There are several studies being carried out in cancer modeling and treatment. Schättler et al. [3] developed a mathematical model with minimal parameters for low-dose metronomic chemotherapy. The model takes into account the angiogenic signaling between the tumor and the vasculature and the tumor inhibitory effects of tumor-immune system interactions. Liu and Yang [4] developed a cancer treatment model with radiotherapy followed by chemotherapy. Jordão and Tavares [5] studied the dynamics of healthy and diseased cells. The model for healthy cells is based on the p53 gene network, cell cycle control mechanism, the MAP kinase pathway, and the PI3K or Akt pathway, a cancer cell model embodied by deregulation in a healthy cell model. Solis-Perez et al. in [6] created a mathematical model that considers the population dynamics among cancer stem cells, tumor cells, healthy cells, the effects of excess estrogen, and the body's natural immune response to cell populations.

Several mathematical models that are more specific to breast cancer have been introduced before. Enderling 
et al. in [7] modeled the breast cancer development, the local treatment, and the recurrence. Furthermore, Prameswari and Adi-Kusumo [8] constructed a model of the phase transition regulation on cell cycle to characterize breast cancer cells' growth in the earlier stage, which is indicated by the anomalies of cell proliferation. The model knows the behavior changes in cell concentration used as an early indicator of cancer growth. In the same year, Kusuma and Adi-Kusumo [9] introduced a mathematical model of DNA damage as the estrogen response involving the G1/S transition phase in the cell cycle. The model is a foundation to understand the initial behavior of breast cancer.

Treatment of breast cancer through chemotherapy can pose a risk to healthy cells throughout the body. The neutrophil is white blood cells that have a short life span (6-10 hours). They provide the first line of defense part of the body's immune system. Neutrophils are the most common types of leukocytes found in human peripheral blood. The neutrophil concentration is about $60 \%$ of white blood cells in human blood. They are major cellular contributors to inflammation, mediating the initial phases of the inflammatory response. They can eliminate microbes through various mechanisms and are essential for the defense of the human body. Acquired neutrophil disorders are usually caused by cytotoxic chemotherapy or immunosuppressive agents. Primary neutrophil disorders usually result from the deactivation of individual gene mutations that result in impaired neutrophil number or function [10].

Neutropenia is defined as a decreasing number of neutrophils in the circulating blood. Chemotherapy-induced neutropenia is one of the most common toxic effects experienced by patients and often threatens the efficiency of chemotherapy use. Neutropenia usually occurs 7 to 10 days after chemotherapy. It can increase the risk of a complication of infection and fever known as febrile neutropenia (FN) [11]. In several papers, they consider the prediction of the occurrence of neutropenia in breast cancer patients. A mathematical model for optimizing neutropenia minimization was introduced in the study by Craig et al. [12]. A model that was developed is based on the characterization of neutrophil production in the bone marrow. The model was integrated with a pharmacokinetic and pharmacodynamic (PKPD) model of a chemotherapy agent. In another case with the model proposed by Craig et al. [12], we will also look at a model of neutrophil interaction with variables that, according to the data, affect the survival of breast cancer patients, namely, lymphocytes, chemotherapy drugs, and albumin.

Lymphocytes are white blood cells that play a role in maintaining the immune system. Lymphocytes are one of the main components of the immune system and are indispensable in the immune response to cancer. Lymphocytes are divided into two types, namely, T lymphocytes and B lymphocytes. T lymphocytes kill bacteria and viruses, while B lymphocytes function to make antibodies that fight antigens. The neutrophillymphocyte ratio (NLR) has been evaluated in various types of cancer. It is proposed as a marker of inflammation to predict outcomes. Increased NLR in the peripheral blood of patients with cancer could be a sign of a manifestation of systemic inflammation induced by cancer cells [14]. Hartono's study [15] discussed the assessment of the neutrophil-lymphocyte and albumin ratio before and after chemotherapy. The results of the study demonstrated a reduction in NLR and an increase in albumin after chemotherapy.

Albumin is the most substantial protein in blood plasma. It is maintaining oncotic pressure, transporting fatty acids to the liver, transporting drugs, shortening the half-life of the drug, transporting bilirubin, binding $\mathrm{Ca} 2+$ ions a buffer solution, transporting thyroid hormones, and transporting other hormones, especially those that can dissolve in fat. Albumin also functions as a transport for various substances, including bilirubin, fatty acids, metals, ions, hormones, and drugs. It plays a vital role in the regeneration and repair process of cells, as an agent that forms cell bonds. Albumin also helps to give signal for the immune system about signs of damage to body cells.

Chemotherapy causes hematopoietic progenitor cells in the bone marrow to experience depletion and acute myelosuppression [16]. In conditions of severe hemopoiesis stress, stem cells can repair themselves, proliferate, and differentiate into various blood cell series in the periphery. However, suppose the self-renewal ability of hemopoiesis stem cells is impaired. In that case, there will be permanent damage to the hematopoietic system resulting in bone marrow failure. It can occur when the bone marrow is exposed to high doses of chemotherapy agents or radiation [17]. The effect of neutropenia appears to be higher than that of cytopenia of the erythroid and platelet series because the composition of neutrophil precursors occupies the most significant number in the bone marrow, which is more than 50\% [18].

In this paper, we consider a new mathematical model on breast cancer therapy that includes the neutropenia effects due to the chemotherapy. Our model is a 4-dimensional system of first-order ODE based on the interaction between the concentrations of neutrophils $(\mathrm{N})$, chemotherapy drugs (C), lymphocytes (L), and albumin (A). We focus our analysis to understand the role of parameters to the steadystate conditions of the system. In this case, we employ bifurcation analysis to study the existence and stability of the equilibria. It is important for calculating the survival level of the breast cancer patients.

\section{Model Formulation}

The interaction between the neutrophils $(\mathrm{N})$, chemotherapy drugs (C), lymphocytes (L), and albumin (A) is shown in Figure 1. The interaction between those substances is created by the results of some medical papers (see [10, 15-18]), and the correlation analysis of the data from National Health and Nutrition Examination Survey (see [14]).

Our model is formulated as follows: 


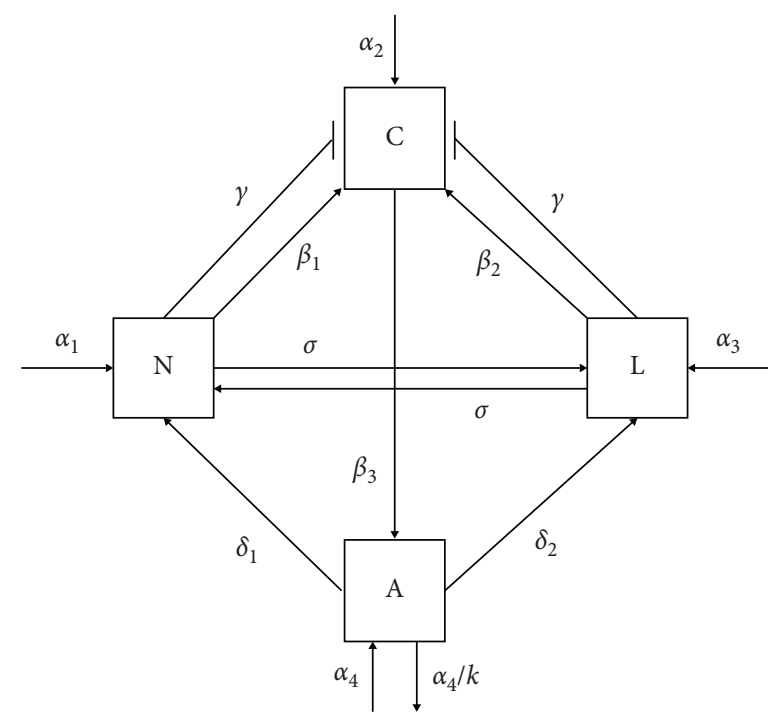

Figure 1: The transfer diagram of substances. The production, degradation, and interaction are denoted as arrow headed line. The inhibition is denoted as blunt arrow.

$$
\begin{aligned}
& \frac{d N}{d t}=\alpha_{1} N-\frac{\sigma N L}{1-\theta N}-\beta_{1} C N+\delta_{1} A N, \\
& \frac{d C}{d t}=\alpha_{2} C-\frac{\gamma C}{N+L} \\
& \frac{d L}{d t}=\alpha_{3} L-\frac{\sigma N L}{1-\theta L}-\beta_{2} C L+\delta_{2} A L \\
& \frac{d A}{d t}=\alpha_{4} A\left(1-\frac{A}{k}\right)+\beta_{3} C A
\end{aligned}
$$

By the transfer diagram shown in Figure 1, we construct a mathematical model that shows the interactions between the neutrophil, lymphocyte, and albumin concentrations due to the appearance of chemotherapy drugs concentration (see system (1)). System (1) is a four- dimensional system of the first-order ODE in indimensional parameter space. The parameters used in the model are assumed to be positive, with the information in Table 1.

\section{Existence of Equilibrium Solutions}

System (1) has eight equilibrium points, but we consider the analysis of the first seven equilibrium points. Calculating the last equilibrium point is very complicated, so we left it as an open problem in this paper. The existences of those equilibrium points are presented in Theorem 1 and Theorem 2.

Theorem 1 (the existences of equilibrium points $T_{1}$ to $T_{6}$ of System (1)).

(i) The equilibrium point $T_{1}=\left(\alpha_{3}\left(-\theta \alpha_{1}+\sigma\right) /\right.$ $\left.-\theta^{2} \alpha_{1} \alpha_{3}+\sigma^{2}, 0, \alpha_{1}\left(-\theta \alpha_{3}+\sigma\right) /-\theta^{2} \alpha_{1} \alpha_{3}+\sigma^{2}, 0\right)$ exists if $\sigma>\theta \alpha_{1}$ and $\sigma>\theta \alpha_{3}$ (ii) The equilibrium points $T_{2}=\left(0, \alpha_{3} / \beta_{2}, \gamma / \alpha_{2}, 0\right)$ and $T_{3}=\left(\gamma / \alpha_{2}, \alpha_{1} / \beta_{1}, 0,0\right)$ exist for all parameter values

(iii) The equilibrium point $T_{4}=\left(\left(\left(k \delta_{1}+\alpha_{1}\right) \theta-\sigma\right)\right.$ $\left(k \delta_{2}+\alpha_{3}\right) /\left(k \delta_{2}+\alpha_{3}\right)\left(k \delta_{1}+\alpha_{1}\right) \theta^{2}-\sigma^{2}, 0, \quad\left(k \delta_{1}+\right.$ $\left.\alpha_{1}\right)\left(\left(k \delta_{2}+\alpha_{3}\right) \theta-\sigma\right) /\left(k \delta_{2}+\alpha_{3}\right)\left(k \delta_{1}+\alpha_{1}\right) \theta^{2}-$ $\left.\sigma^{2}, k\right)$ exists if it satisfies the conditions in Table 2

(iv) The equilibrium point $T_{5}=\left(0,-\left(k \delta_{2}+\alpha_{3}\right) \alpha_{4} /\right.$ $\left.k \beta_{3} \delta_{2}-\alpha_{4} \beta_{2}, \gamma / \alpha_{2},-k\left(\alpha_{3} \beta_{3}+\alpha_{4} \beta_{2}\right) / k \beta_{3} \delta_{2}-\alpha_{4} \beta_{2}\right)$ exists if $k \beta_{3} \delta_{2}<\alpha_{4} \beta_{2}$

(v) The equilibrium point $T_{6}=\left(\gamma / \alpha_{2},-\left(k \delta_{1}+\alpha_{1}\right) \alpha_{4} /\right.$ $\left.k \beta_{3} \delta_{1}-\alpha_{4} \beta_{1}, 0,-k\left(\alpha_{1} \beta_{3}+\alpha_{4} \beta_{1}\right) / k \beta_{3} \delta_{1}-\alpha_{4} \beta_{1}\right)$ exists if $k \beta_{3} \delta_{1}<\alpha_{4} \beta_{1}$

Theorem 2 (the existences of equilibrium points $T_{7}$ of System (1)). The equilibrium point $T_{7}\left(N_{7}, C_{7}, L_{7}, A_{7}\right)$ with $C_{7}= \pm \sqrt{w}\left(\theta \alpha_{1}-\sigma\right)+\left(\begin{array}{ll}\left(2 \alpha_{2} x+y\right) & \left.\alpha_{1}-2 x \gamma \sigma\right) \theta-\sigma y / \beta_{1} \theta\end{array}\right.$ $\left(2 \alpha_{2} x \pm \sqrt{w}+y\right)$ and $N_{7}=- \pm \sqrt{w}+y / 2 \alpha_{2} \theta x$, $L_{7}=$ $2 \gamma \theta x \pm \sqrt{w}+y / 2 \alpha_{2} \theta x, A_{7}=0$ where $w=\gamma^{2} \theta^{4} z^{2}-4 \gamma \alpha_{2} \theta^{3}$ $z^{2}+\theta^{2}\left(4 \alpha_{2}^{2} z^{2}+2 \gamma \sigma \alpha_{2} \quad\left(\beta_{1}-\beta_{2}\right) z+4 \gamma^{2} \sigma^{2} \beta_{1} \beta_{2}\right)-8 \alpha_{2} \sigma \theta$ $\left(\alpha_{2}\left(\beta_{1}-\beta_{2}\right) z / 2+\gamma \sigma \beta_{1} \beta_{2}\right)+\sigma^{2} \alpha_{2}^{2}\left(\beta_{1}+\beta_{2}\right)^{2}, \quad x=\theta \alpha_{1} \beta_{2}-$ $\theta \alpha_{3} \beta_{1}+\sigma \beta_{1}-\sigma \beta_{2}, \quad y=-\gamma \theta^{2} \alpha_{1} \beta_{2}+\gamma \theta^{2} \alpha_{3} \beta_{1}+2 \gamma \sigma \theta \beta_{2}-$ $\sigma \alpha_{2} \beta_{1}-\sigma \alpha_{2} \beta_{2}$, and $z=-\alpha_{1} \beta_{2}+\alpha_{3} \beta_{1}$ has two possible existences due to value $\pm \sqrt{w}$. If $\sqrt{w}$ value is negative, then condition of existence is $-\sigma(2 \gamma \theta x-\sqrt{w}+y) / \theta\left(-2 \alpha_{2} x+\right.$ $\sqrt{w}-y)<\alpha_{1}<-\sigma(2 \gamma \theta x-\sqrt{w}+y) / \theta\left(-2 \alpha_{2} x+\sqrt{w}-y\right)$, $w>0, x>0, y>0$. If $\sqrt{w}$ value is positive, then condition of existence is $(2 \gamma \theta x+\sqrt{w}+y) \sigma / \theta \quad\left(2 \alpha_{2} x+\sqrt{w}+y\right)<\alpha_{1}<$ $(2 \gamma \theta x+\sqrt{w}+y) \sigma / \theta\left(2 \alpha_{2} x+\sqrt{w}+y\right), w>0, x>0, y>0$.

Then, the results of Theorem 1 are summarized in Table 3 in the appendix. Based on the results of the theorem, we show the area in parameter spaces that two or more equilibria coexist in Table 4. 
TABle 1: Parameters description.

\begin{tabular}{lcc}
\hline Parameter & Description \\
\hline$\alpha_{1}$ & Neutrophil production rate & Unit \\
$\alpha_{2}$ & Chemotherapy drugs absorbing rate & $1 /$ day \\
$\alpha_{3}$ & Lymphocyte production rate & $1 /$ day \\
$\alpha_{4}$ & Albumin production rate & $1 /$ day \\
$\beta_{1}$ & Neutrophil reaction rate due to chemotherapy regimens & $1 / \% /$ day \\
$\beta_{2}$ & Lymphocyte reaction rate due to chemotherapy regimens & $1 / \% /$ day \\
$\beta_{3}$ & Albumin reaction rate due to chemotherapy regimen & $1 / \% /$ day \\
$\delta_{1}$ & Albumin and neutrophils reaction rate & $1 / \% /$ day \\
$\delta_{2}$ & Albumin and lymphocytes reaction rate & $1 / \% /$ day \\
$\sigma$ & Neutrophils and lymphocytes reaction rate & $\% /$ day \\
$\gamma$ & Decay of chemotherapy regimens & $\% /$ day \\
$\theta$ & Leucocyte saturation parameter & $\%$ \\
$k$ & Maximum capacity of albumin in blood plasma & $\%$ \\
\hline
\end{tabular}

TABLE 2: Terms of existences $T_{4}$.

\begin{tabular}{lc}
\hline No. & Terms of existences \\
\hline 1 & $\alpha_{1}<-\delta_{1} \theta k-\sigma / \theta, \alpha_{3}<-\delta_{2} \theta k-\sigma / \theta, \delta_{1}<\sigma / k \theta, \delta_{2}<\sigma / k \theta$ \\
2 & $\alpha_{3}<-\delta_{1} \delta_{2} \theta^{2} k^{2}-\sigma^{2} / k \theta^{2} \delta_{1}, \delta_{1}<\sigma / k \theta, \delta_{2}<\sigma / k \theta,-\delta_{1} \theta k-\sigma / \theta<\alpha_{1},-\delta_{2} \theta k-\sigma / \theta<\alpha_{3}$ \\
3 & $\alpha_{3}=-\delta_{1} \delta_{2} \theta^{2} k^{2}-\sigma^{2} / k \theta^{2} \delta_{1}, \delta_{1}<\sigma / k \theta, \delta_{2}<\sigma / k \theta,-\delta_{1} \theta k-\sigma / \theta<\alpha_{1}$ \\
4 & $\delta_{1}<\sigma / k \theta, \delta_{2}<\sigma / k \theta,-\delta_{1} \delta_{2} \theta^{2}-\sigma^{2} / k \theta^{2} \delta_{1}<\alpha_{3},-\delta_{1} \theta k-\sigma / \theta<\alpha_{1}$ \\
5 & $\delta_{1}=\sigma / k \theta, \delta_{2}<\sigma / k \theta,-\delta_{2} \theta k-\sigma / \theta<\alpha_{3}$ \\
6 & $\delta_{1}<\sigma^{2} / \delta_{2} \theta^{2} k^{2}, \delta_{2}<\sigma / k \theta, \sigma / k \theta<\delta_{1},-\delta_{2} \theta k-\sigma / \theta<\alpha_{3}$ \\
7 & $\delta_{1}=\sigma^{2} / \delta_{2} \theta^{2} k^{2}, \delta_{2}<\sigma / k \theta,-\delta_{2} \theta k-\sigma / \theta<\alpha_{3}$ \\
8 & $\delta_{2}<\sigma / k \theta, \sigma^{2} / \delta_{2} \theta^{2} k^{2}<\delta_{1},-\delta_{2} \theta k-\sigma / \theta<\alpha_{3}$ \\
9 & $\delta_{2}=\sigma / k \theta, \alpha_{3}<-\sigma\left(\delta_{1} \theta k-\sigma\right) / \delta_{1} \theta^{2} k, \delta_{1}<\sigma / k \theta,-\delta_{1} \theta k-\sigma / \theta<\alpha_{1}$ \\
10 & $\alpha_{3}=-\sigma\left(\delta_{1} \theta k-\sigma\right) / \delta_{1} \theta^{2} k, \delta_{2}=\sigma / k \theta, \delta_{1}<\sigma / k \theta,-\delta_{1} \theta k-\sigma / \theta<\alpha_{1}$ \\
11 & $\delta_{2}=\sigma / k \theta, \delta_{1}<\sigma / k \theta,-\delta_{1} \theta k-\sigma / \theta<\alpha_{1},-\sigma\left(\delta_{1} \theta k-\sigma\right) / \delta_{1} \theta^{2} k<\alpha_{3}$ \\
12 & $\delta_{1}=\sigma / k \theta, \delta_{2}=\sigma / k \theta$ \\
13 & $\delta_{2}=\sigma / k \theta, \sigma / k \theta<\delta_{1}$ \\
14 & $\delta_{3}<-\delta_{1} \delta_{2} \theta^{2} k^{2}-\sigma^{2} / k \theta^{2} \delta_{1}, \delta_{1}<\sigma^{2} / \delta_{2} \theta^{2} k^{2}, \sigma / k \theta<\delta_{2},-\delta_{1} \theta k-\sigma / \theta<\alpha_{1}$ \\
15 & $\alpha_{3}=-\delta_{1} \delta_{2} \theta^{2} k^{2}-\sigma^{2} / k \theta^{2} \delta_{1}, \delta_{1}<\sigma^{2} / \delta_{2} \theta^{2} k^{2}, \sigma / k \theta<\delta_{2},-\delta_{1} \theta k-\sigma / \theta<\alpha_{1}$ \\
16 & $\delta_{1}<\sigma^{2} / \delta_{2} \theta^{2} k^{2}, \sigma / k \theta<\delta_{2},-\delta_{1} \theta k-\sigma / \theta<\alpha_{1},-\delta_{1} \delta_{2} \theta^{2} k^{2}-\sigma^{2} / k \theta^{2} \delta_{1}<\alpha_{3}$ \\
17 & $\delta_{1}=\sigma^{2} / \delta_{2} \theta^{2} k^{2}, \sigma / k \theta<\delta_{2}, \sigma\left(\delta_{2} \theta k-\sigma\right) / \delta_{2} \theta^{2} k<\alpha_{1}$ \\
18 & $\delta_{1}<\sigma / k \theta, \sigma / k \theta<\delta_{2}, \sigma^{2} / \delta_{2} \theta^{2} k^{2}<\delta_{1},-\delta_{1} \theta k-\sigma / \theta<\alpha_{1}$ \\
19 & $\delta_{1}=\sigma / k \theta, \sigma / k \theta<\delta_{2}$ \\
20 & $\sigma / k \theta<\delta_{1}, \sigma / k \theta<\delta_{2}$ \\
\hline
\end{tabular}

TABLE 3: The existences of equilibrium points.

\begin{tabular}{|c|c|}
\hline Equilibrium points $T_{n}\left(N_{n}, C_{n}, L_{n}, A_{n}\right)$ & $\begin{array}{l}\text { Terms of } \\
\text { existences }\end{array}$ \\
\hline$T_{1}=\left(\alpha_{3}\left(-\theta \alpha_{1}+\sigma\right) /-\theta^{2} \alpha_{1} \alpha_{3}+\sigma^{2}, 0, \alpha_{1}\left(-\theta \alpha_{3}+\sigma\right) /-\theta^{2} \alpha_{1} \alpha_{3}+\sigma^{2}, 0\right)$ & $\begin{array}{l}\sigma>\theta \alpha_{1} \text { and } \\
\sigma>\theta \alpha_{2}\end{array}$ \\
\hline $\begin{array}{l}T_{2}=\left(0, \alpha_{3} / \beta_{2}, \gamma / \alpha_{2}, 0\right) \\
T_{3}=\left(\gamma / \alpha_{2}, \alpha_{1} / \beta_{1}, 0,0\right)\end{array}$ & $\begin{array}{l}\text { Always exist } \\
\text { Always exist }\end{array}$ \\
\hline $\begin{array}{l}T_{4}=\left(\left(\left(k \delta_{1}+\alpha_{1}\right) \theta-\sigma\right)\left(k \delta_{2}+\alpha_{3}\right) /\left(k \delta_{2}+\alpha_{3}\right)\left(k \delta_{1}+\alpha_{1}\right) \theta^{2}-\sigma^{2}, 0,\left(k \delta_{1}+\alpha_{1}\right)\right. \\
\left.\left(\left(k \delta_{2}+\alpha_{3}\right) \theta-\sigma\right) /\left(k \delta_{2}+\alpha_{3}\right)\left(k \delta_{1}+\alpha_{1}\right) \theta^{2}-\sigma^{2}, k\right)\end{array}$ & Table 2 \\
\hline $\begin{array}{l}T_{5}=\left(0,-\left(k \delta_{2}+\alpha_{3}\right) \alpha_{4} / k \beta_{3} \delta_{2}-\alpha_{4} \beta_{2}, \gamma / \alpha_{2},-k\left(\alpha_{3} \beta_{3}+\alpha_{4} \beta_{2}\right) / k \beta_{3} \delta_{2}-\alpha_{4} \beta_{2}\right) \\
T_{6}=\left(\gamma / \alpha_{2},-\left(k \delta_{1}+\alpha_{1}\right) \alpha_{4} / k \beta_{3} \delta_{1}-\alpha_{4} \beta_{1}, 0,-k\left(\alpha_{1} \beta_{3}+\alpha_{4} \beta_{1}\right) / k \beta_{3} \delta_{1}-\alpha_{4} \beta_{1}\right)\end{array}$ & $\begin{array}{l}k \beta_{3} \delta_{2}<\alpha_{4} \beta_{2} \\
k \beta_{3} \delta_{1}<\alpha_{4} \beta_{1}\end{array}$ \\
\hline
\end{tabular}

TABle 4: Coexistence of the equilibrium points $T_{1}$ to $T_{6}$ of System (1).

\begin{tabular}{lc}
\hline The area of coexistence & Equilibrium \\
\hline$\left\{\sigma \mid \delta_{1} k \theta \geq \sigma>\theta \alpha_{1} \wedge \delta_{2} k \theta \geq \sigma>\theta \alpha_{3}, \delta_{1}<\alpha_{4} \beta_{1} / k \beta_{3}, \delta_{2}<\alpha_{4} \beta_{2} / k \beta_{3}, \sigma \in \mathscr{R}\right\}$ & $T_{1}, T_{2}, T_{3}, T_{4}, T_{5}, T_{6}$ \\
$\left\{\sigma \mid \delta_{1} k \theta \geq \sigma>\theta \alpha_{1} \wedge \delta_{2} k \theta \geq \sigma>\theta \alpha_{3}, \quad \delta_{1} \geq \alpha_{4} \beta_{1} / k \beta_{3}, \delta_{2}<\alpha_{4} \beta_{2} / k \beta_{3}, \quad \sigma \in \mathscr{R}\right\}$ & $T_{1}, T_{2}, T_{3}, T_{4}, T_{5}$ \\
$\left\{\sigma \mid \delta_{1} k \theta \geq \sigma>\theta \alpha_{1} \wedge \delta_{2} k \theta \geq \sigma>\theta \alpha_{3}, \delta_{1}<\alpha_{4} \beta_{1} / k \beta_{3}, \delta_{2} \geq \alpha_{4} \beta_{2} / k \beta_{3}, \quad \sigma \in \mathscr{R}\right\}$ & $T_{1}, T_{2}, T_{3}, T_{4}, T_{6}$ \\
$\left\{\sigma \mid \delta_{1} k \theta \geq \sigma>\theta \alpha_{1} \wedge \delta_{2} k \theta \geq \sigma>\theta \alpha_{3}, \quad \sigma \in \mathscr{R}\right\}$ & $T_{1}, T_{2}, T_{3}, T_{4}$ \\
$\left\{\sigma \mid \sigma>\theta \alpha_{1} \wedge \sigma>\theta \alpha_{3}, \quad \sigma \in \mathscr{R}\right\}$ & $T_{1}, T_{2}, T_{3}$ \\
$\{\sigma \mid \sigma \in \mathscr{R}\}$ & $T_{1}, T_{2}$ \\
\hline
\end{tabular}

${ }^{*}$ means that the condition for the existence of $T_{4}$ is number 20 in Table 2. 


\section{Stability Analysis}

We apply the linearization method to determine the stability of the equilibrium point of System (1), and we have the Jacobian matrix as follows:

$$
J(T)=\left[\begin{array}{cccc}
j_{11} & -\beta_{1} N & \frac{\sigma N}{\theta N-1} & \delta_{1} N \\
\frac{\gamma C}{(L+N)^{2}} & j_{22} & \frac{\gamma C}{(L+N)^{2}} & 0 \\
\frac{\sigma L}{L \theta-1} & -\beta_{2} L & j_{33} & \delta_{2} L \\
0 & \beta_{3} A & 0 & j_{44}
\end{array}\right],
$$

where $\quad j_{11}=\alpha_{1}+\sigma L / \theta N-1-\sigma N L \theta / \quad(\theta N-1)^{2}-\beta_{1} C+$ $\delta_{1} A, \quad j_{22}=\alpha_{2}-\gamma / L+N, \quad j_{33}=\alpha_{3}+\sigma N / L \theta-1-$ $\sigma N L \theta /(L \theta-1)^{2}-\beta_{2} C+\delta_{2} A$, and $j_{44}=\left(\beta_{3} C+\quad \alpha_{4}\right) k-$ $2 \alpha_{4} A / k$.

The eigenvalues can be obtained from the characteristic equation $|J(T)-\lambda I|=0$ evaluated at each point of equilibrium. The stability analysis discussed in this study is only at the equilibrium point $T_{1}$ to $T_{6}$. The stability analysis of the equilibrium point will be carried out by considering the numerical simulation in special cases. The stability analysis of the equilibrium point T4 will be carried out by considering the numerical simulation in special cases.

4.1. Equilibrium Point $T_{1}$. Linearization of System (1) near the equilibrium $T_{1}$ produces the characteristic equation as follows: $a b\left(-\alpha_{4}+\lambda\right) /\left(\left(\alpha_{1}+\alpha_{3}\right) \sigma-2 \alpha_{3} \alpha_{1} \theta\right)\left(-\theta \alpha_{3}+\sigma\right)\left(-\theta \alpha_{1}+\sigma\right) \sigma^{2}=0$,

where $a=\gamma \sigma^{2}+\left(\alpha_{1}+\alpha_{3}\right)\left(\lambda-\alpha_{2}\right) \sigma-\theta \alpha_{1} \alpha_{3}\left(\gamma \theta+2 \lambda-2 \alpha_{2}\right)$ and $\quad b=\left(\lambda^{2}-\alpha_{1} \alpha_{3}\right) \sigma^{4}-\left(-\alpha_{3} \alpha_{1}^{2}+\left(\lambda^{2}-2 \lambda \alpha_{3}-\alpha_{3}^{2}\right) \alpha_{1}+\right.$ $\left.\lambda^{2} \alpha_{3}\right) \theta \sigma^{3}+\theta^{2} \lambda \alpha_{1} \alpha_{3} \quad\left(\lambda-2 \alpha_{1}-2 \alpha_{3}\right) \sigma^{2}+\theta^{3} \alpha_{1}\left(\left(\lambda-\alpha_{3}\right) \alpha_{1}^{2}-\right.$ $\left.\alpha_{3}^{2} \alpha_{1}+\lambda \alpha_{3}^{2}\right) \alpha_{3} \sigma+\theta^{4} \alpha_{1}^{3} \alpha_{3}^{3}$.

From (3), it can be seen that there is a positive eigenvalue, i.e., $\lambda_{1}=\alpha_{4}$. It can be concluded that the equilibrium point $T_{1}$ is unstable.

4.2. Equilibrium Point $T_{2}$. The characteristic equation of the linearization of System (1) near the equilibrium point $T_{2}$ is $-\left(\left(\left(\lambda-\alpha_{1}\right) \alpha_{2}+\quad \gamma \sigma\right) \beta_{2}+\beta_{1} \alpha_{3} \alpha_{2}\right)\left(\lambda^{2}+\alpha_{2} \alpha_{3}\right)\left(\left(\alpha_{4}-\lambda\right) \beta_{2}+\right.$ $\left.\alpha_{3} \beta_{3}\right) / \alpha_{2} \beta_{2}^{2}=0$, so that its eigenvalues are $\lambda_{1}=\sqrt{-\alpha_{2} \alpha_{3}}, \lambda_{2}=$ $-\sqrt{-\alpha_{2} \alpha_{3}}, \lambda_{3}=\alpha_{3} \beta_{3}+\alpha_{4} \beta_{2} / \beta_{2}$, and $\lambda_{4}=-\gamma \sigma \beta_{2}-\alpha_{1} \beta_{2} \alpha_{2}+$ $\beta_{1} \alpha_{3} \alpha_{2} / \alpha_{2} \beta_{2}$. In this case, we have a positive definite eigenvalue, so the equilibrium point of $T_{2}$ is unstable.

4.3. Equilibrium Point $T_{3}$. The characteristic equation of the linearization of System (1) near the equilibrium point $J_{3}$ is $-\left(\lambda^{2}+\alpha_{1} \alpha_{2}\right) \quad\left(\left(\alpha_{4}-\lambda\right) \beta_{1}+\alpha_{1} \beta_{3}\right) \quad\left(\left(\left(\lambda-\alpha_{3}\right) \alpha_{2}+\gamma \sigma\right) \beta_{1}+\right.$ $\left.\alpha_{1} \beta_{2} \alpha_{2}\right) / \alpha_{2} \beta_{1}^{2}=0$ where the eigenvalues are $\lambda_{1}=\sqrt{-\alpha_{1} \alpha_{2}}, \lambda_{2}=-\sqrt{-\alpha_{1} \alpha_{2}}, \lambda_{3}=\alpha_{1} \beta_{3}+\alpha_{4} \beta_{1} / \beta_{1}$, and $\lambda_{4}=$ $\lambda=-\gamma \sigma \beta_{1}+\alpha_{1} \beta_{2} \alpha_{2}-\beta_{1} \alpha_{3} \alpha_{2} / \alpha_{2} \beta_{1}$. The positive eigenvalues indicate that the equilibrium point of $T_{3}$ is unstable.

4.4. Equilibrium Point $T_{4}$. The Jacobian matrix obtained from the linearization of System (1) near the equilibrium point $T_{4}$ is

$$
J_{4}=\left[\begin{array}{cccc}
-\frac{d e}{f}\left(1+\frac{e}{\sigma}\right) & -\frac{\beta_{1} e d}{(e+f) \sigma+e f} & -\frac{d e}{f} & \frac{\delta_{1} e d}{(e+f) \sigma+e f} \\
0 & -\frac{\gamma((e+f) \sigma+e f)}{-\sigma(d+c)+2 d e+2 \sigma d}+\alpha_{2} & 0 & 0 \\
-\frac{c f}{e} & -\frac{\beta_{2} c f}{(e+f) \sigma+e f} & -\frac{d f}{e}\left(1+\frac{e}{\sigma}\right) & \frac{c \delta_{2} f}{(e+f) \sigma+e f} \\
0 & \beta_{3} k & 0 & -\alpha_{4}
\end{array}\right],
$$

where $\quad c=k \delta_{1}+\alpha_{1}, d=k \delta_{2}+\alpha_{3}, \quad e=\theta c-\sigma$, and $f=$ $\theta d-\sigma$. It is assumed that the values of $c, d, e$, and $f$ are positive. The characteristic equation formed is

$$
\frac{g h\left(\alpha_{4}+\lambda\right)}{\sigma^{2} f(\sigma c-2 d e-\sigma d) e}=0,
$$

where $g=\left(-c \lambda \sigma+c \sigma \alpha_{2}+2 d e \lambda-2 d e \alpha_{2}+d \lambda \sigma-d \sigma \alpha_{2}+\right.$ $e f \gamma+e \gamma \sigma+f \gamma \sigma) \quad$ and $h=\left(c d \sigma^{2} f e-d^{2} e^{3} f-\right.$
$2 d^{2} e^{2} f \sigma-d^{2} e f \sigma^{2}-d e^{3} \lambda \sigma-e^{2} d \lambda \quad \sigma^{2}-d e f^{2} \lambda \sigma-$ $\left.d f^{2} \lambda \sigma^{2}-e f \lambda^{2} \sigma^{2}\right)$

which has the eigenvalues $\lambda_{1}=-\alpha_{4}, \lambda_{2,3}=-\left(d e^{3}+\right.$ $\left.d e^{2} \sigma+d e f^{2}+d f^{2} \sigma \pm \sqrt{m} / e f \sigma\right)$ where $m=4 c d e^{2} f^{2} \sigma^{2}+$ $d^{2} e^{6}+2 d^{2} e^{5} \sigma-2 d^{2} e^{4} f^{2}+d^{2} e^{4} \sigma^{2}-4 d^{2} e^{3} f^{2} \sigma+d^{2} e^{2} f^{4}-$ $2 d^{2} e^{2} f^{2} \sigma^{2}+2 d^{2} e f^{4} \sigma+d^{2} f^{4} \sigma^{2} \quad$ and $\quad \lambda_{4}=\alpha_{2}+e f \gamma+$ $e \gamma \sigma+f \gamma \sigma / \sigma c-2 d e-\sigma d$. Based on the equations of $e, f$, and $\lambda_{4}$, the stability conditions for the equilibrium point of 
$T_{4}$ are obtained, namely, $\theta c>\sigma, \theta d>\sigma$, and $\alpha_{2}<e f \gamma+e \gamma \sigma+f \gamma \sigma / \sigma c-2 d e-\sigma d$.

4.5. Equilibrium Point $T_{5}$. Linearization of System (1) near the equilibrium point $T_{5}$ obtains the characteristic equation as follows:

$$
\begin{aligned}
\lambda^{4}+ & \lambda^{3}\left(-\frac{q}{o \alpha_{2}}+\frac{n \alpha_{4}}{o}\right)+\lambda^{2}\left(-\frac{n \alpha_{4} q}{\alpha_{2} o^{2}}+\frac{\beta_{2} \alpha_{4} p \alpha_{2}}{o}\right) \\
& +\lambda\left(-\frac{\alpha_{4} \beta_{2} p q}{o^{2}}+\frac{\alpha_{2} \alpha_{4} p n}{o}\right)-\frac{\alpha_{4} p n q}{o^{2}}=0,
\end{aligned}
$$

where $\quad n=\alpha_{3} \beta_{3}+\quad \alpha_{4} \beta_{2}, o=\alpha_{4} \beta_{2}-k \beta_{3} \delta_{2}$, and $p=k \delta_{2}+$ $\alpha_{3}, q=\alpha_{2}\left(k \delta_{1} n-\beta_{1} \alpha_{4} p+o \alpha_{1}\right)-\gamma o \sigma$, all of which are assumed to be positive. The terms of the stability of this point are analyzed using the Routh-Hurwitz criteria, and we obtain

$$
\begin{aligned}
& 0<-\frac{\alpha_{4} p n q}{o^{2}}, \\
& 0<\frac{\alpha_{4} n \alpha_{2}-q}{o \alpha_{2}}, \\
& 0<-\frac{n\left(o p\left(-\alpha_{4} \beta_{2}+o\right) \alpha_{2}^{3}+n q \alpha_{2} \alpha_{4}-q^{2}\right) \alpha_{4}}{\alpha_{2} o^{2}\left(\alpha_{4} n \alpha_{2}-q\right)}, \\
& 0<\frac{p\left(-\alpha_{4} \beta_{2}+o\right)\left(n o^{2} p \alpha_{2}^{4} \alpha_{4}-o p q \alpha_{2}^{3} \alpha_{4} \beta_{2}+n q^{2} \alpha_{4} \alpha_{2}-q^{3}\right)}{\left(o p\left(-\alpha_{4} \beta_{2}+o\right) \alpha_{2}^{3}+n q \alpha_{2} \alpha_{4}-q^{2}\right) o^{2}} .
\end{aligned}
$$

4.6. Equilibrium Point $T_{6}$. Linearization of System (1) near the equilibrium $T_{6}$ produces the characteristic equation as follows:

$$
\begin{aligned}
\lambda^{4} & +\lambda^{3}\left(-\frac{v}{\alpha_{2} s}+\frac{r \alpha_{4}}{s}\right)+\lambda^{2}\left(-\frac{r \alpha_{4} v}{\alpha_{2} s^{2}}+\frac{\alpha_{4} u \beta_{1} \alpha_{2}}{s}\right) \\
& +\lambda\left(-\frac{\alpha_{4} u \beta_{1} v}{s^{2}}+\frac{\alpha_{4} u r \alpha_{2}}{s}\right)-\frac{\alpha_{4} u r v}{s^{2}}=0,
\end{aligned}
$$

where $\quad r=\alpha_{1} \beta_{3}+\alpha_{4} \beta_{1}, s=-\quad k \beta_{3} \delta_{1}+\alpha_{4} \beta_{1}, u=k \delta_{1}+$ $\alpha_{1}$, and $v=\alpha_{2}\left(k \delta_{2} r-\alpha_{4} \beta_{2} u+\alpha_{3} s\right)-\gamma s \sigma$, all of which are assumed to be positive. The terms of the stability of this point are analyzed using the Routh-Hurwitz criteria with the result as follows:

$$
\begin{aligned}
& 0<-\frac{\alpha_{4} u r v}{s^{2}}, \\
& 0<\frac{r \alpha_{4} \alpha_{2}-v}{\alpha_{2} s}, \\
& 0<-\frac{r\left(s u\left(-\alpha_{4} \beta_{1}+s\right) \alpha_{2}^{3}+r v \alpha_{2} \alpha_{4}-v^{2}\right) \alpha_{4}}{\alpha_{2} s^{2}\left(r \alpha_{4} \alpha_{2}-v\right)}, \\
& 0<\frac{u\left(-\alpha_{4} \beta_{1}+s\right)\left(r s^{2} u \alpha_{2}^{4} \alpha_{4}-s u v \alpha_{2}^{3} \alpha_{4} \beta_{1}+r v^{2} \alpha_{2} \alpha_{4}-v^{3}\right)}{\left(s u\left(-\alpha_{4} \beta_{1}+s\right) \alpha_{2}^{3}+r v \alpha_{2} \alpha_{4}-v^{2}\right) s^{2}} .
\end{aligned}
$$

The stability of the equilibrium points $T_{2}$ and $T_{5}$ is very important for neutropenia. If those equilibria are stable, the neutrophils will decrease and go to zero. This situation triggers the neutropenia case due to the chemotherapy. The other important equilibrium point is $T_{6}$. The stability of this equilibrium shows the reduction of lymphocyte which is one of the main component in the immune system against the cancer.

\section{Numerical Simulations}

Before treatment, all breast cancer patients will perform a complete blood laboratory examination, including examining the concentration of neutrophils, lymphocytes, and albumin. If the patient is indicated that there is breast cancer, then the patient will be treated medically through chemotherapy. However, chemotherapy drugs also consider the side effects of neutropenia. Mathematical models are needed to understand and to anticipate the side effects of neutropenia. Simulations of the changes in neutrophils, chemotherapy drugs, lymphocytes, and albumin concentration during chemotherapy are performed to predict neutropenia.

The simulation was carried out based on the assumption that the concentration of neutrophils was 0.8 (80\%) and that of lymphocytes was $0.2(20 \%)$ from white blood cells, and that of albumin was 0.7 (70\%) from blood plasma. Chemotherapy concentration is assumed to be 0.4 (40\%) from the maximum limit capacity of chemotherapy drugs in the body. Medically, neutrophils, albumin, and neutrophils in human blood circulation cannot be zero, so the first case used in this simulation is directed towards the equilibrium point of $T_{4}$. By using the parameter values $\alpha_{1}=0.3, \alpha_{2}=$ $0.3, \alpha_{3}=0.2, \alpha_{4}=0.33, \beta_{1}=0.7, \beta_{2}=0.6, \beta_{3}=0.75, \sigma=$ $0.53, \theta=0.95, k=0.8, \delta_{1}=0.6, \delta_{2}=0.6$, and $\gamma=0.9$, we obtained dynamic changes in the model as shown in the curves of Figures 2 and 3. Numerical simulations are also used to verify the results of the dynamic analysis that has been carried out. Based on the predetermined parameter values, using four different initial conditions, the solution will go to the equilibrium point $T_{4}$, as shown in Figure 4 .

Figures 2 and 3 show the dynamic changes of the four observed variables, where neutrophils at the beginning of chemotherapy increased until the second day and then decreased significantly to equilibrium at 0.725 . It is consistent with the medical facts of the side effects of neutropenia after chemotherapy. The chemotherapy concentration at the beginning of chemotherapy is 0.4 , gradually decreasing until it reaches zero. Albumin plays a role in forming white blood cells; in this case, the albumin has increased to 0.96 before dropping to 0.8 on day 25 . The increase in albumin correlates with an increase in lymphocytes. Lymphocytes that play a role against cancer cells and chemotherapy have increased to 0.456 . Based on these dynamic changes, it is known that the neutrophillymphocyte ratio has decreased, where at the beginning of chemotherapy, it has a ratio of 4 . After chemotherapy, it drops to 1.6.

The conditions in Figures 2 and 3 show the occurrence of neutropenia, where the neutrophil concentration is 


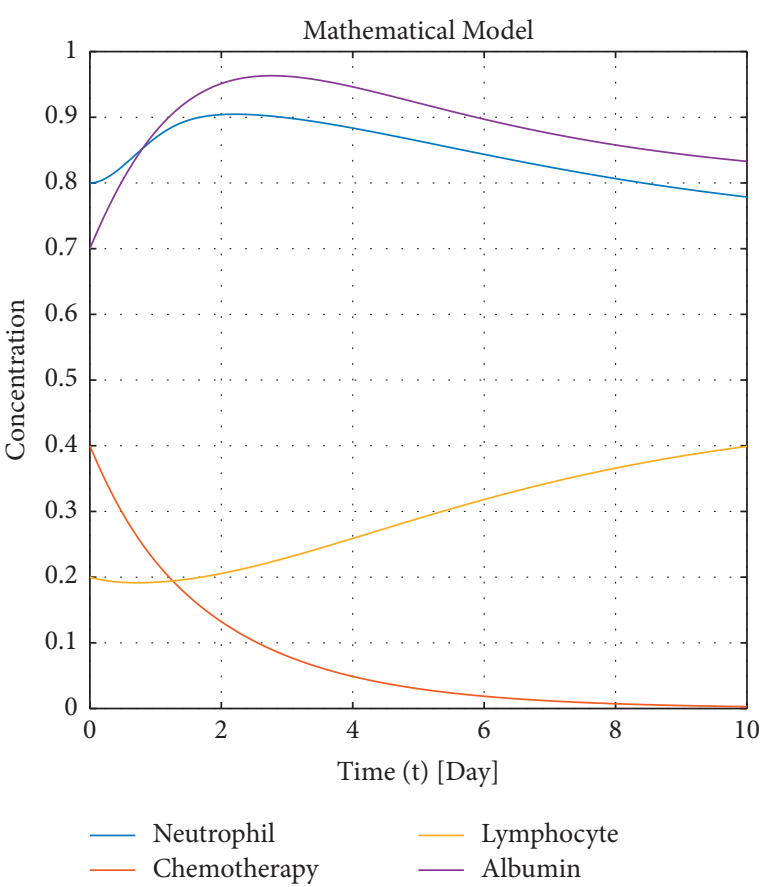

Figure 2: The dynamics of change in N, C, L, and A over time until $t=10$ in the first case.

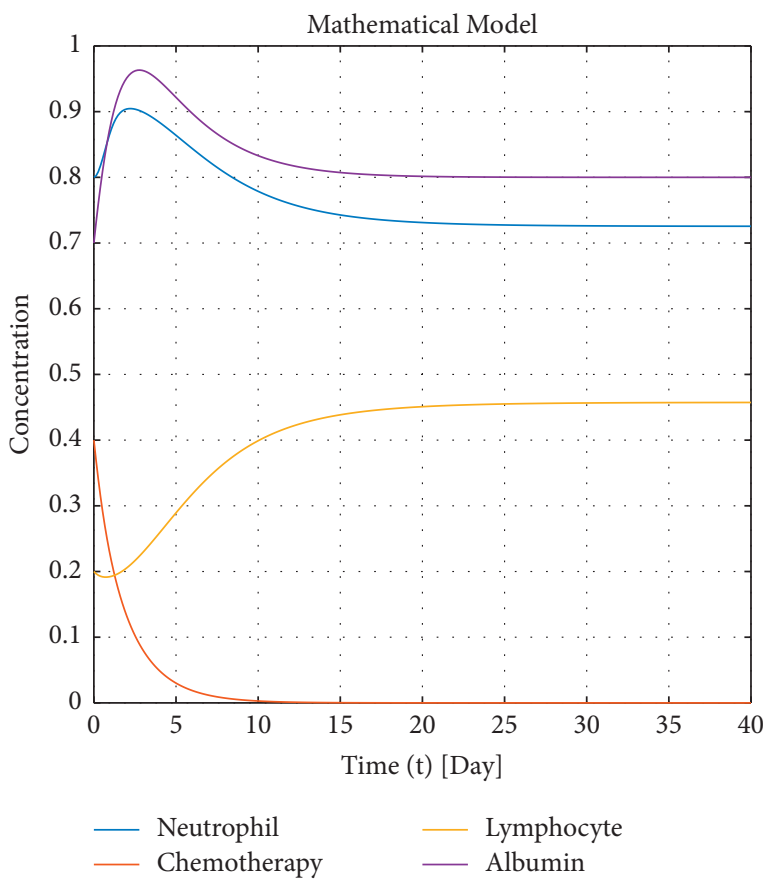

Figure 3: The dynamics of change in N, C, L, and A over time until $t=40$ in the first case.

significantly reduced. One alternative to prevent neutropenia is to reduce albumin. In the second case, we take the same parameter value as first case for $\alpha_{1}, \alpha_{2}, \alpha_{3}, \alpha_{4}$, $\beta_{1}, \beta_{2}, \beta_{3}, \sigma, \theta, \delta_{1}, \delta_{2}$, and $\gamma$, by changing $k$ value. Albumin can be reduced by reducing its maximum capacity $(k)$ to 0.68 so that the albumin concentrates towards the steady-state at this value. In this case, the neutrophils raised at the

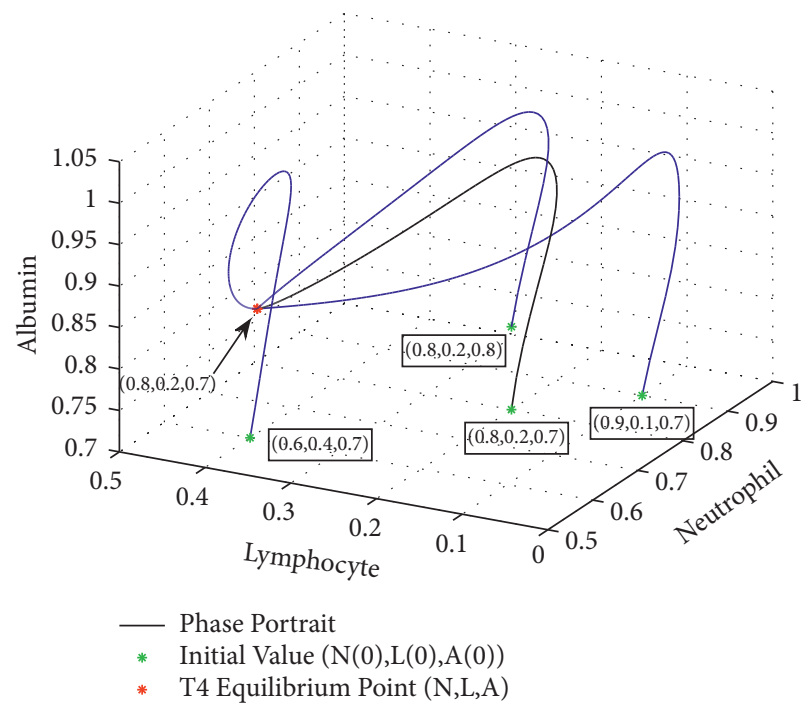

Figure 4: Phase portrait of the system solution with four different initial conditions.

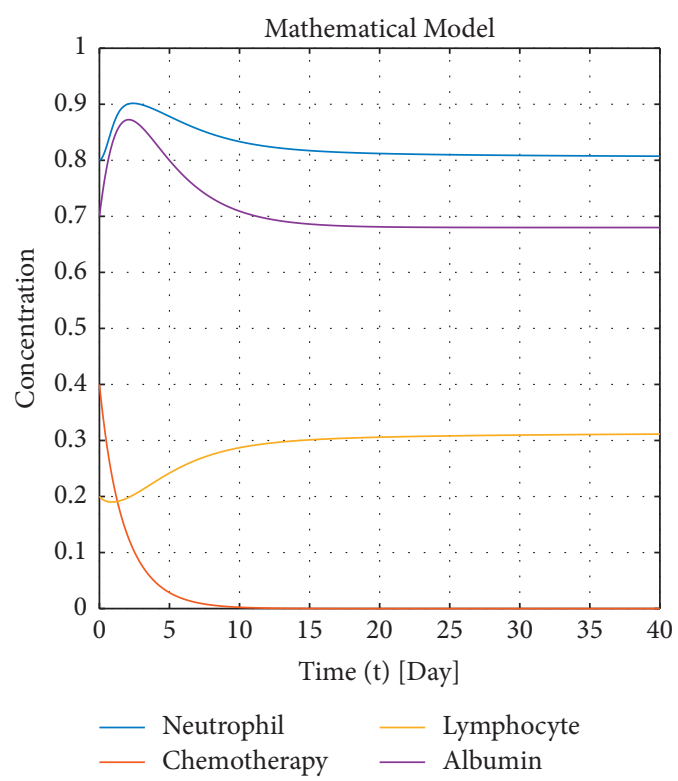

Figure 5: The dynamics of change in N, C, L, and A over time in the second case.

beginning of chemotherapy will not decrease significantly, and it reaches the steady-state, which is at 0.8 . The lymphocytes continued to increase towards the final state at 0.31 . The neutrophil-lymphocyte ratio in this second case was reduced to 2.58 after chemotherapy. This second case simulation is shown in the graph in Figure 5.

In this section, we can find dynamic simulations of the interaction of blood components, represented by neutrophils, lymphocytes, and albumin, with the effects of chemotherapy drugs. Based on a mathematical model simulation, it can be seen that the regulation of albumin concentration in blood has a role in preventing and predicting the side effects of neutropenia due to chemotherapy 
drugs. So, if the patient's condition requires chemotherapy treatment, medical experts can reduce the level of albumin concentration in the blood. It is an alternative solution to prevent neutropenia based on a mathematical approach.

\section{Concluding Remarks}

Breast cancer is a dangerous disease with the side effects of treatment. A side effect of chemotherapy for breast cancer that needs to be watched out for is neutropenia, where the neutrophils in the white blood cells are reduced to being abnormal. The dynamics of the three components of blood cells with chemotherapy concentrations have been constructed using a differential equation system (1) based on data and medical facts described previously.

Simulations are carried out to determine changes in the number of neutrophils, chemotherapy, lymphocytes, and albumin concentrations from time to time if given the initial conditions. By knowing the dynamics that will occur, we can control changes in the concentration of the three components of blood cells to return to normal after chemotherapy. This study formed a dynamic model of blood components and chemotherapy based on hospital data and medical literature.

The mathematical model constructed in this study can describe the dynamics of changes in the concentration of neutrophils, chemotherapy, lymphocytes, and albumin in the patient's body to predict the possibility of neutropenia. One alternative in preventing neutropenia based on the model in this study is to reduce the albumin concentration. The results of this study can be used as an alternative solution for treating neutropenia in breast cancer patients, in mathematical point of view.

\section{Data Availability}

No data were used to support this study.

\section{Conflicts of Interest}

The authors declare that they have no conflicts of interest.

\section{Acknowledgments}

The authors thank the Deputy for Research and Strengthening Development of the Ministry of Research and Technology Indonesia (the National Research and Innovation Agency) which has provided research funding through Penelitian Dasar Unggulan Perguruan Tinggi (PDUPT) with contract agreement number 1683/UN1/DITLIT/DIT-LIT/PT/2021. The authors also thank the Department of Mathematics, Faculty of Mathematics and Natural Sciences, Universitas Gadjah Mada for the support in research facilities.

\section{References}

[1] Globocan, Cancer today: international agency for research on cancer, https://gco.iarc.fr/today/data/factsheets/cancers/20Breast-fact-sheet.pdf, 2018.

[2] M. C. Oz and M. F. Roizen, You: The Owner's Manual: An Insider's Guide to the Body that Will Make You Healthier and Younger, Harper Collins, New York, NY, USA, 2013.
[3] H. Schättler, U. Ledzewicz, and B. Amini, "Dynamical properties of A minimally parameterized mathematical model for metronomic chemotherapy," Journal of Mathematical Biology, vol. 72, no. 5, pp. 1255-1280, 2016.

[4] Z. Liu and C. Yang, "A mathematical model of cancer treatment by radiotherapy followed by chemotherapy," Mathematics and Computers in Simulation, vol. 124, pp. 1-15, 2016.

[5] G. Jordão and J. N. Tavares, "Mathematical models in cancer therapy," Biosystems, vol. 162, pp. 12-23, 2017.

[6] J. E. Solís-Pérez, J. F. Gómez-Aguilar, and A. Atangana, “A fractional mathematical model of breast cancer competition model," Chaos, Solitons \& Fractals, vol. 127, pp. 38-54, 2019.

[7] H. Enderling, M. A. J. Chaplain, A. R. A. Anderson, and J. S. Vaidya, "A mathematical model of breast cancer development, local treatment and recurrence," Journal of Theoretical Biology, vol. 246, no. 2, pp. 245-259, 2007.

[8] K. Prameswari and F. Adi-Kusumo, "A mathematical model for phase transition regulation on the cell cycle in early stage of breast cancer," in AIP Conference Proceedingsvol. 2192, no. 1, AIP Publishing LLC, Article ID 060014, 2019.

[9] M. F. Kusuma and F. Adi-Kusumo, "A mathematical modelling for estradiol influence on DNA damage response and G1/S transition phase regulations in early stage of breast cancer," in AIP Conference Proceedingsvol. 2192, no. 1, AIP Publishing LLC, Article ID 060012, 2019.

[10] R. Suzuki, X. Wei, P. K. Allen, J. D. Cox, R. Komaki, and S. H. Lin, "Prognostic significance of total lymphocyte count, neutrophil-to-lymphocyte ratio, and platelet-to-lymphocyte ratio in limited-stage small-cell lung cancer," Clinical Lung Cancer, vol. 20, no. 2, pp. 117-123, 2018.

[11] E. A. Lundqvist, "Principles of chemotherapy," International Journal of Gynecology \& Obstetrics, vol. 119, pp. S151-S154, Article ID 119S2, 2012.

[12] M. Craig, A. R. Humphries, F. Nekka, J. Bélair, J. Li, and M. C. Mackey, "Neutrophil dynamics during concurrent chemotherapy and G-CSF administration: mathematical modelling guides dose optimisation to minimise neutropenia," Journal of Theoretical Biology, vol. 385, pp. 77-89, 2015.

[13] H. K. Lehman and B. H. Segal, "The role of neutrophils in host defense and disease," The Journal of Allergy and Clinical Immunology, vol. 145, no. 6, pp. 1535-1544, 2020.

[14] National Health and Nutrition Examination Survey, Complete Blood Count with 5-part Differential-Whole Blood (CBC_G), National Bureau of Economic Research, Cambridge, MA, USA, 2014, http://data.nber.org/nhanes/2011-2012/CBC_G.htm.

[15] B. Hartono, V. S. Pontoh, and M. A. Merung, "Penilaian jumlah neutrofil, limfosit dan trombosit, kadar protein reaktif C, kadar albumin, rasio neutrofil limfosit, serta rasio trombosit limfosit sebelum dan setelah terapi pada penderita karsinoma payudara," Jurnal Biomedik: JBM, vol. 73 pages, 2015.

[16] V. T. Devita Jr, S. Hellman, and S. A. Rosenberg, Principles and Practise of Oncologypp. 1595-1634, Lippincot Williams and Wilkins, Philadelphia, PA, USA, 5th edition, 2008.

[17] K. R. Georgiou, B. K. Foster, and C. J. Xian, "Damage and recovery of the bone marrow microenvironment induced by cancer chemotherapy - potential regulatory role of chemokine CXCL12/receptor CXCR4 signalling," Current Molecular Medicine, vol. 10, no. 5, pp. 440-453, 2010.

[18] F. Crea, E. Giovannetti, P. L. Zinzani, and R. Danesi, "Pharmacologic rationale for early G-CSF prophylaxis in cancer patients and role of pharmacogenetics in treatment optimization," Critical Reviews in Oncology, vol. 72, no. 1, pp. 21-44, 2009. 